\title{
NWPEsSe: an Adaptive-Learning Global Optimization Algorithm for Nanosized Cluster Systems
}

Jun Zhang, Vassiliki-Alexandra Glezakou, ${ }^{*}$ Roger Rousseau, Manh-Thuong Nguyen

$†$ Physical Sciences Division, Pacific Northwest National Laboratory (PNNL), Richland, WA, 99352, United States

* Corresponding author: Vanda.Glezakou@pnnl.gov

\section{Contents}

Section S1

Figure $\mathrm{S1}$

Section S2

Section S3
Solving Thomson's Problem

Plot of the Potential eq. (4).

Energy Decomposition of Au8GrD-2 and Au8GrD-3

NWPEsSe as a Structure Generator 
Section S1 Solving Thomson's Problem

Thomson's problem ${ }^{1}$ is defined as looking for the minimum electrostatic potential energy configuration of electrons constrained to a unit sphere. Thus, for an $\mathrm{N}$-electron system, Thomson's problem aims to minimize the following function:

$$
T\left(\mathbf{r}_{1}, \mathbf{r}_{2}, \cdots, \mathbf{r}_{N}\right)=\sum_{i<j} \frac{1}{r_{i j}}
$$

Since all $\mathbf{r}_{i}$ 's are constrained on a unit sphere, thus they can be further parametrized as

$$
\mathbf{r}_{i}=\left(\cos \theta_{i} \cos \phi_{i}, \cos \theta_{i} \sin \phi_{i}, \sin \theta_{i}\right)
$$

Now $T$ becomes a $2 N$-variable function. Given an arbitrary initial guess, $T$ can be optimized with respect to $\theta_{i}$ 's and $\phi_{i}$ 's using the limited-memory-Broyden-Fletcher-Goldfarb-Shanno (L-BFGS) algorithm. ${ }^{2}$

Figure S1 Plot of the potential eq. (4). It is continuously differentiable thus presents no problems in the local optimization. In NWPEsSe, $k$ is (arbitrarily) set to $50000 \mathrm{kcal} \mathrm{nm}^{-2}$.

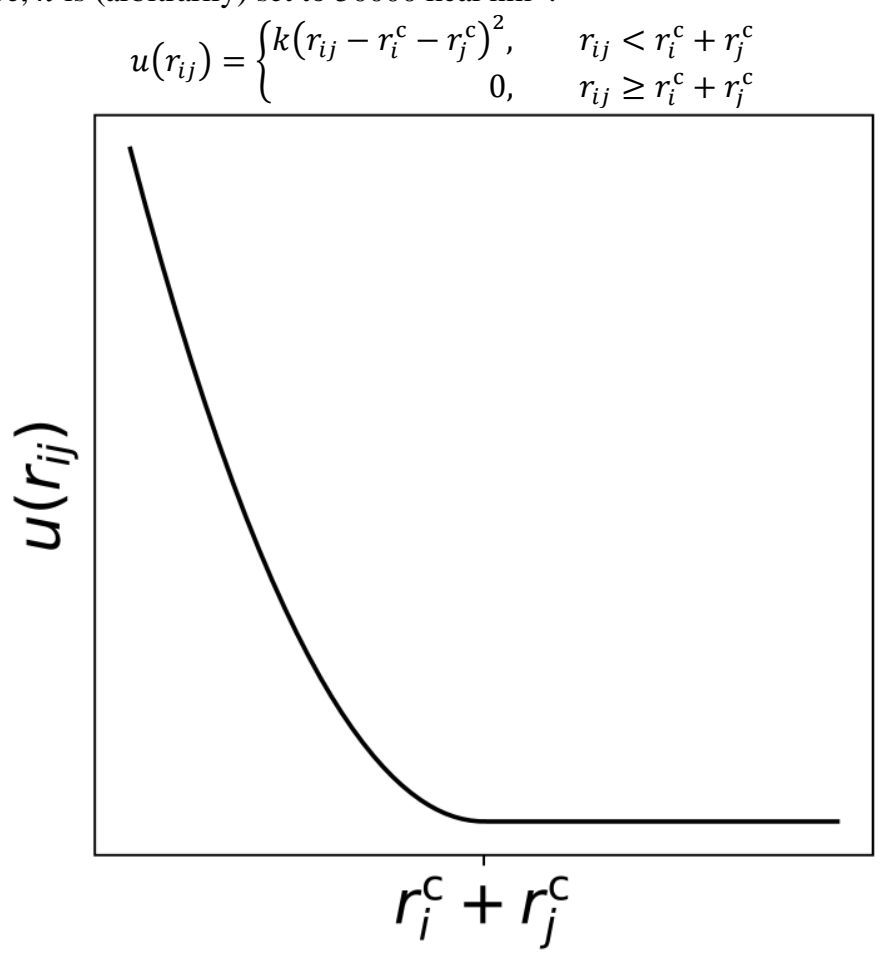


Section S2 Energy Decomposition of Au8GrD-2 and Au8GrD-3

The energy difference at PBE-D3 level of theory

$$
\Delta E=E[\text { Au8GrD-3 }]-E[\text { Au8GrD-2 }]=+2.48 \mathrm{kcal} \mathrm{mol}^{-1}
$$

is given in the text. This energy can be decomposed into two physically meaningful terms:

The deformation energy difference is defined as:

$$
\Delta E=\Delta E_{\text {deform }}+\Delta E_{\text {inter }}
$$

$$
\Delta E_{\text {deform }}=E_{\text {deform }}[\mathrm{Au} 8]+E_{\text {deform }}[\mathrm{GrD}]
$$

$$
E_{\text {deform }}[\mathrm{Au} 8]=(E[\text { Au8 in Au8GrD-3 }]-E[\text { Au8 in Au8GrD-2] })
$$

$E_{\text {deform }}[\mathrm{GrD}]=(E[\mathrm{GrD}$ in Au8GrD-3 $]-E[$ GrD in Au8GrD-2] $)$

Here, $E$ [Au8 in Au8GrD-3]/E[Au8 in Au8GrD-2] is the energy of $\mathrm{Au}_{8}$ at its geometry in Au8GrD-3/Au8GrD-2, thus $E_{\text {deform }}[\mathbf{A u 8}]$ is the deformation energy of $\mathrm{Au}_{8}$ from $\mathbf{A u 8 G r D - 2}$ to Au8GrD-3. For $E_{\text {deform }}[\mathrm{GrD}]$ similar definition applies.

The interaction energy difference is defined as:

$$
\begin{gathered}
\Delta E_{\text {inter }}=E_{\text {inter }}[\text { Au8GrD-3 }]-E_{\text {inter }}[\text { Au8GrD-2 }] \\
E_{\text {inter }}[\text { Au8GrD-3 }]=E[\text { Au8GrD-3 }]-E[\text { Au8 in Au8GrD-3 }]-E[\text { GrD in Au8GrD-3 }] \\
E_{\text {inter }}[\text { Au8GrD-2 }]=E[\text { Au8GrD-2 }]-E[\text { Au8 in Au8GrD-2 }]-E[\text { GrD in Au8GrD-2 }]
\end{gathered}
$$

Thus, eqs. (S9) and (S10) are pure interaction energies.

These terms were calculated to be

$$
\begin{aligned}
& E_{\text {deform }}[\text { Au8 }]=+5.85 \mathrm{kcal} \mathrm{mol}^{-1} \\
& E_{\text {deform }}[\mathrm{GrD}]=+1.35 \mathrm{kcal} \mathrm{mol}^{-1} \\
& E_{\text {inter }}[\text { Au8GrD-3 }]=-86.97 \mathrm{kcal} \mathrm{mol}^{-1} \\
& E_{\text {inter }}\left[\text { Au8GrD-2] }=-82.25 \mathrm{kcal} \mathrm{mol}^{-1}\right.
\end{aligned}
$$

Then we can calculate $\Delta E_{\text {deform }}=+7.20 \mathrm{kcal} \mathrm{mol}^{-1}$ and $\Delta E_{\text {inter }}=-4.72 \mathrm{kcal} \mathrm{mol}^{-1}$. Therefore, when Au8-0 and Au8-1 land on GrD without corruption (Au8GrD-2 and Au8GrD-3), it is the pure interaction energy between gold cluster and graphene oxide that reduces their energy difference. 
Section S3 NWPEsSe as a Structure Generator

When no quantum chemistry program is used, NWPEsSe can generate clusters using given structural features by itself. Although the generated structures cannot be ranked by energy, they are already very reasonable for quantum chemistry calculations or molecular dynamics simulations, thanks to the relaxation by optimizing eq. (5). Below are three examples.

(1) Given $\mathrm{H}_{2} \mathrm{O}$ and $\mathrm{C}_{6} \mathrm{H}_{5} \mathrm{CC} \equiv \mathrm{H}$, NWPEsSe can generate clusters in a box as a mixture or a bi-phase one.
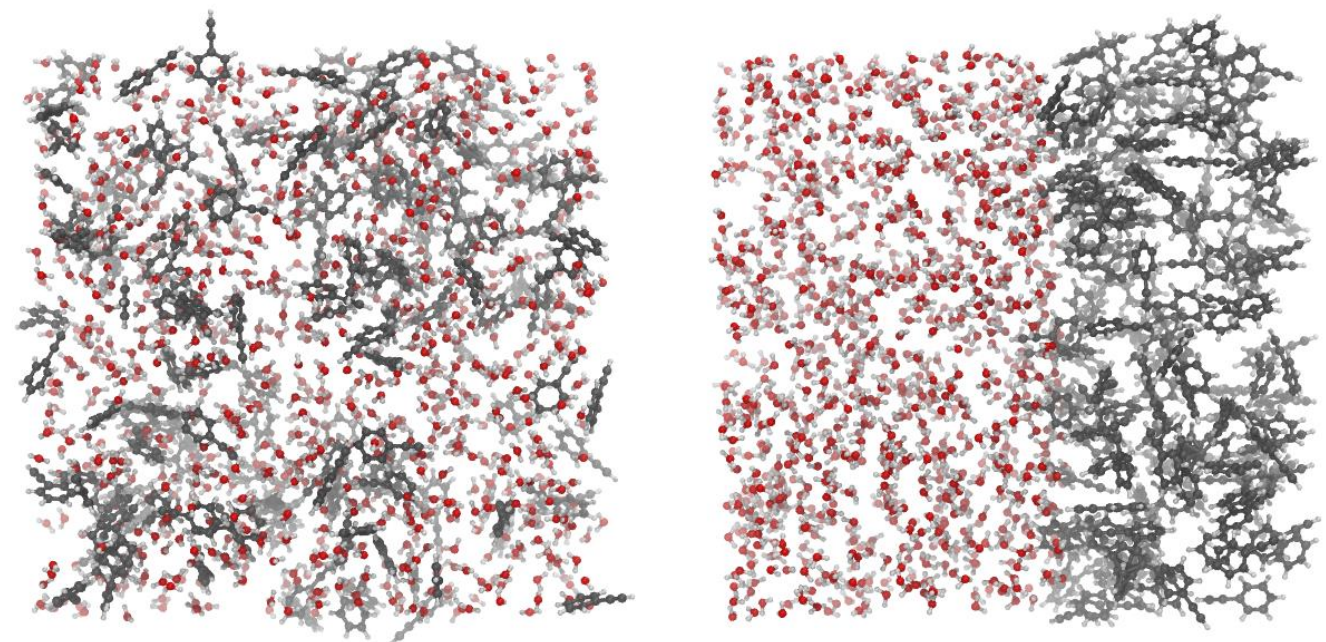

(2) Given $\mathrm{H}_{2} \mathrm{O}$ and phosphatidylcholine (POPC) molecules, NWPEsSe can generate reversed micelle or layer structures.
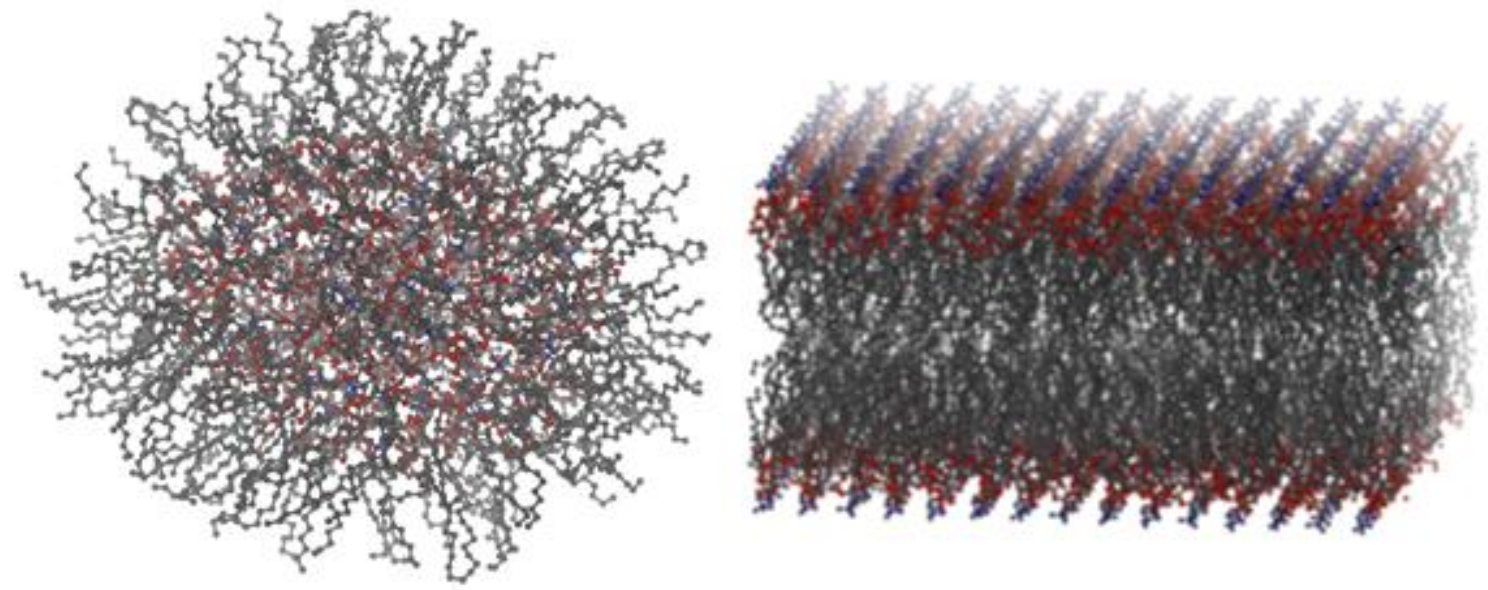

(3) Given a $\mathrm{Ni}_{38}$ slab and $36 \mathrm{CO}$ molecules, NWPEsSe can put CO randomly or evenly on the surface.
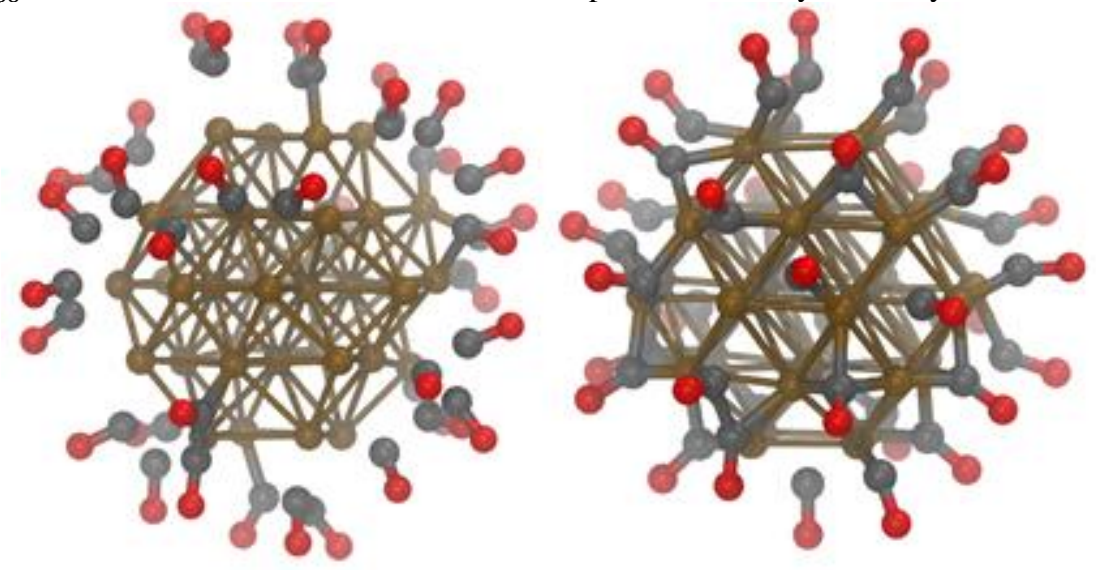

NWPEsSe provides interfaces to other programs. If quantum chemistry calculations are non-affordable, it is also possible to connect NWPEsSe with some molecular dynamics programs, like Lammps or Gromacs, to carry out local optimizations. See NWPEsSe Manual for details. 


\section{References}

1 Thomson, J. J., XXIV. On the Structure of the Atom: an Investigation of the Stability and Periods of Oscillation of a Number of Corpuscles Arranged at Equal Intervals around the Circumference of a Circle; with Application of the Results to the Theory of Atomic Structure. Philos. Mag. 1904, 7 (39), 237-265.

2. Liu, D. C.; Nocedal, J., On the Limited Memory BFGS Method for Large Scale Optimization. Math. Program. 1989, 45 (1), 503-528. 